\title{
AMENAZAS Y RIESGOS SOCIONATURALES CANTÓN MONTECRISTI: BASES DEL PLAN DE ORDENAMIENTO Y DESARROLLO TERRITORIAL.
}

Guerrero, Omar Antonio ${ }^{1 *}$; Camargo Mora, María Gabriela ${ }^{2}$, Baque-Solís, Byron Simón ${ }^{3}$, Guerrero-Camargo, Omar Alejandro ${ }^{4}$

${ }^{1}$ Docente de la Facultad de Ingeniería - Escuela de Ingeniería Geológica. Universidad de Los Andes - Mérida. Grupo de Ciencias de la Tierra TERRA.

${ }^{2}$ Docente del Departamento de Arquitectura - Ordenamiento Territorial. Universidad Técnica Particular de Loja - Ecuador. Grupo TEYPI.

${ }^{3}$ Docente Universidad Laica Eloy Alfaro de Manabí.

${ }^{4}$ Universidad de los Andes. Faculta de Ingeniería - Escuela de Civil. Mérida - Venezuela. Ingeniero Empresa Habit, CA. San Luis Potosí - México.

* Autor para correspondencia: oag2021@gmail.com

Recibido: 2020/09/29

Aprobado: 2020/11/17

DOI: https://doi.org/10.26621/XVI23.2020.12.A08.PUCESI.2550.6684

\section{RESUMEN}

La Planificación Territorial analiza el territorio de manera vinculante, global e integral, tanto en el ámbito local como en el regional; se presenta como una oportunidad para generar políticas públicas orientadas a la mitigación de los riesgos de desastres y la adaptación al cambio climático, entre otros elementos. En el cantón de Montecristi se orientan estudios a la comprensión de las amenazas multilineales y riesgos socionaturales. Se plantea una metodología integrada que involucra el estudio geomecánico del suelo urbano, las variables hidroclimáticas y las condiciones sísmicas del cantón, siguiendo el protocolo de la Secretaría Nacional de gestión de Riesgos y Emergencia, así como la normativa de Planifica Ecuador, con el objetivo de orientar el componente estructurante del plan de desarrollo y ordenamiento territorial del cantón de Montecristi. En virtud de los resultados, se ha reconocido al cantón como una zona muy susceptible a inundaciones y a la amenaza sísmica.

Palabras clave: riesgos socionaturales, desarrollo urbano, controles geológicos

\section{ABSTRACT}

Territorial Planning analyzes the territory in a binding, global and comprehensive way, both locally and regionally, it is presented as an opportunity to generate public policies aimed at mitigating disaster risks and adapting to climate change, among other elements In the Montecristi canton, studies are aimed at understanding multilinear threats and socio-natural risks. An integrated methodology is proposed that involves the geomechanical study of the urban land, the hydroclimatic variables and the seismic conditions of the canton, following the the National Secretariat protocol for Risk and Emergency Management, as well as the regulations of Planifica Ecuador, with the objective to guide the structuring component of the development plan and territorial ordering of the Montecristi canton. By virtue of the results, the canton has been recognized as an area very sustheceptible to floods and seismic threats.

Keywords: socio-natural risks, urban development, geological control 


\section{INTRODUCCIÓN}

La evaluación de las amenazas naturales en el cantón de Montecristi consiste en el análisis del control geológico, sísmico e hidroclimáticos, para determinar el potencial de inundaciones, movimientos en masa y licuación de suelos (Cruden y Varnes, 1996; BID, 2019 y SNGRE, 2019), con la finalidad de obtener una sectorización de riesgos a través de la aplicación de la metodología del PMA-GSA (2009), SNGRE (2019) y BID (2019), modificada y adaptada a las condiciones de la geológicas del cantón. Las diferentes etapas incluyen la investigación y caracterización de las amenazas naturales, especialmente la de naturaleza hidrogeomorfológica y sísmicas más representativas (Gómez y Salcedo, 1987), así como las evaluaciones de los estudios de mecánica de suelos, todos ellos fundamentales para el desarrollo de las actividades y acciones dentro del PDOT y PUGS (Camargo, 2010; Guerrero y Benavides, 2020); dichos elementos permiten, además, definir con precisión las restricciones, orientaciones, limitantes y potencialidades de los usos urbanos actuales y potenciales sostenibles.

Las variables que conforman el componente biofísico del emplazamiento urbano del cantón Montecristi presentan condiciones favorables para el emplazamiento de zonas urbanas con actividades potenciales de turismo y actividad comercial y residencial (GAD, 2016), pero también existen condiciones desfavorables por encontrarse ciertos espacios bajo amenaza sísmica y de inundación por lluvias excepcionales, lo que se presenta como condicionante para el desarrollo de las áreas urbano-rurales. Por tanto, su crecimiento debe garantizar calidad de vida a la población, al mismo tiempo que se mitigue la exposición a las amenazas y vulnerabilidad de la población.

\section{Emplazamiento de la ciudad}

El cantón Montecristi se encuentra ubicado en $1^{\circ} 2^{\prime} 37^{\prime \prime}$, latitud sur, y $80^{\circ} 39^{\prime}$ de longitud oeste. Posee una extensión territorial de 734,2 km2; forma parte de la provincia de Manabí, limita por el norte con los cantones Manta y Jaramijó, al sur con el cantón Jipijapa y el Océano Pacífico, por el este con los Cantones Portoviejo y Jipijapa y oeste por el Océano Pacífico (Figura 1). Está dividido en cinco parroquias urbanas (Montecristi, Aníbal San Andrés, Gral. Eloy Alfaro, Colorado, Leónidas Proaño) y una parroquia rural, La Pila (INEC, 2010), con una población proyectada de 107785 habitantes (GAD, 2016).

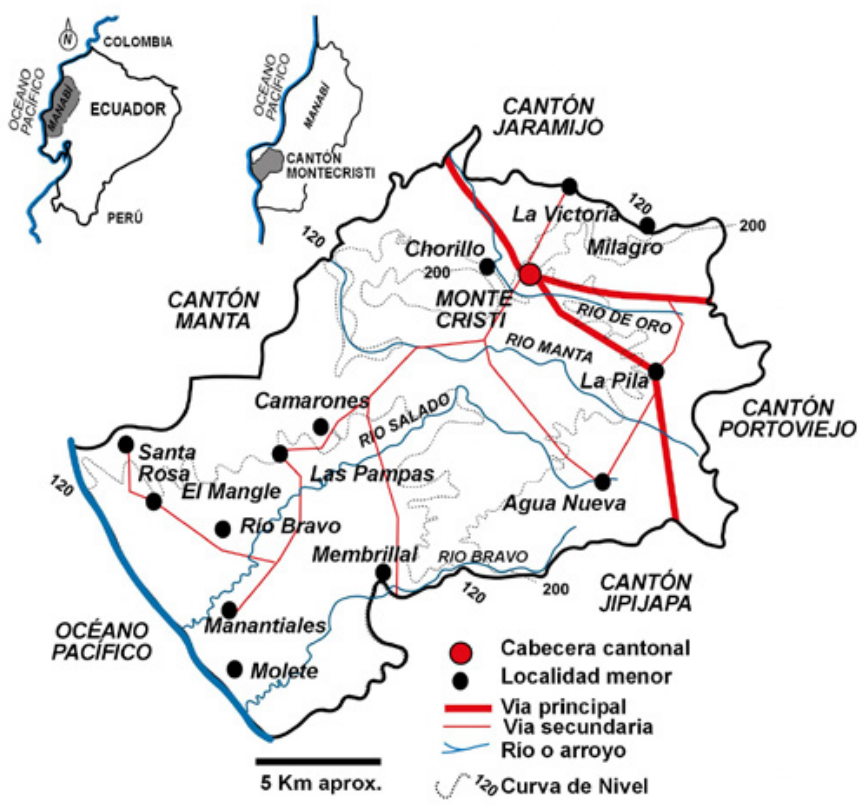

Figura 1. Asentamientos humanos principales del cantón Montecristi.

\section{MATERIALES Y MÉTODOS}

Se realiza un estudio de caso, donde se aplica procedimiento de valoración de variables fisiconaturales, especialmente clima, geología (litología), pendientes críticas y saturación de suelos, entre otras, para determinar amenazas y peligrosidad en cuencas hidrográficas y zonas urbanas a través de los lineamientos del Proyecto Multinacional Andino (2009), Secretaría Nacional de Gestión de Riesgos y Emergencias (2019) y Planifica Ecuador (2019), que permitan determinar la susceptibilidad a inundaciones, movimientos de masa y exposición a la sismicidad. Finalmente, se realiza una correlación y análisis integral de la geomecánica de suelos de la ciudad de Montecristi, empleando perforaciones de suelos realizados por la empresa INGENNYA - NYLIC (2013), bajo las normas ASTM1586 (2001) y NEC-11 (2019).

\section{RESULTADOS Y DISCUSIÓN}

\section{Condiciones geológicas - geomorfológicas del cantón Monte- cristi}

En el territorio del cantón Montecristi, se reconocen siete unidades geológicas, todas correspondientes al Mioceno y Cuaternario (INIGEMM, 2020) que ocupan $94,7 \%$ del territorio; solo un 5,3\% corresponden a mantos basáltico-cretácicos (Figura 2). Además, se reconoce pliegues estructurales que dominan el relieve, formando un gran sinclinal en la zona central del territorio, donde se produce el alineamiento de la microcuenca del río Salado, así como un anticlinal localizado al norte del cantón y fallamiento geológico local con dirección este-oeste con abundante fallas menores en paralelos con dirección norte-sur.

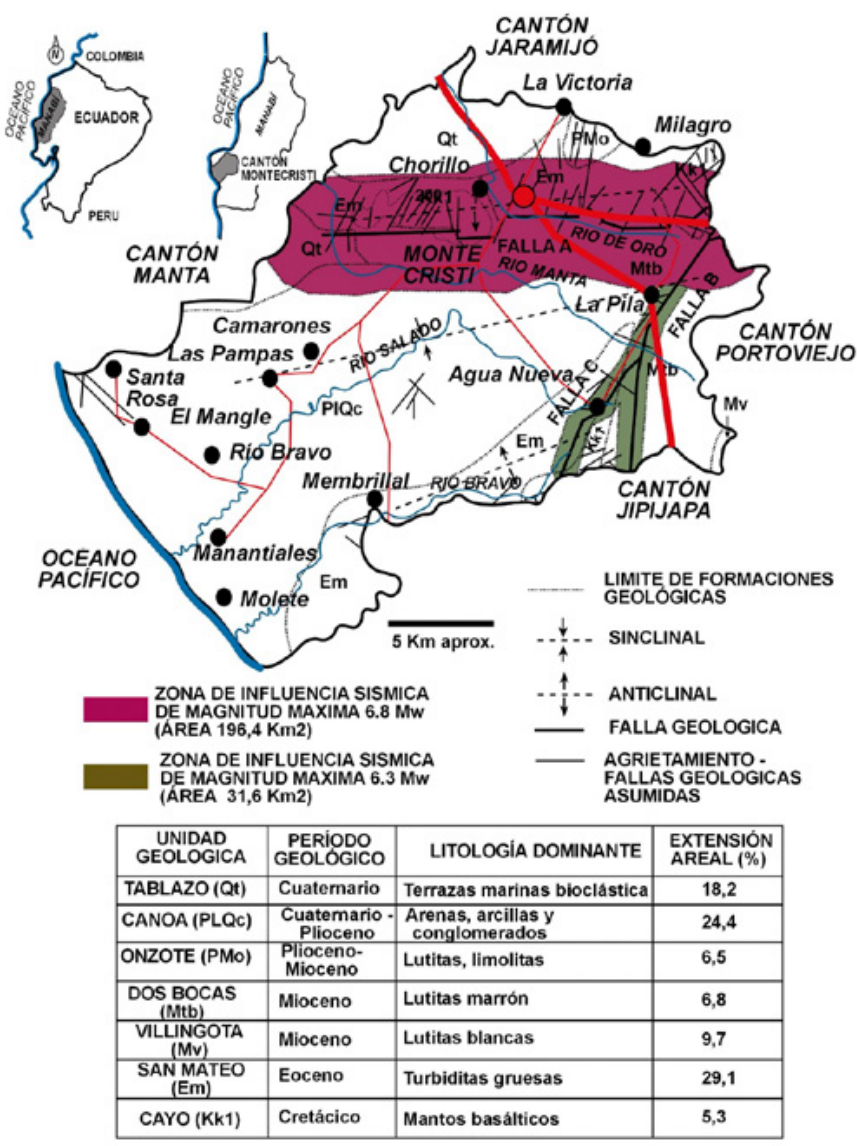

Figura 2. Geología del cantón Montecristi. Fuente: INIGEMM (2020).

Hacia la parte noreste del cantón, específicamente en la zona donde se emplaza la ciudad de Montecristi, se reconocen estructuras geológicas 
tipo anticlinal fallado, con eje en dirección sureste - noroeste; la ciudad de Montecristi se localiza hacia el flanco norte de este alto estructural.

\section{Geomorfología regional}

El cantón Montecristi posee un relieve característico de la región costera, con una topografía de sistemas de colinas medias (antiguo volcán - cerro Montecristi - 640 msnm) y lomas de bajas (>300 msnm) a muy bajas, que fluyen hacia amplios fondos de valles fluviales, interrumpidas por elevaciones de hasta $80 \mathrm{msnm}$. Sobre este relieve piedemonte-costero se localizan los principales centros poblados urbanos como son: Montecristi, Aníbal San Andrés, Gral. Eloy Alfaro, Colorado, Leónidas Proaño, entre otros.

Tres grandes unidades geomorfológicas se describen en el relieve del espacio costero - llanura aluvial del cantón Montecristi (Figura 3): Sistemas de piedemonte colinas y lomeríos medios y altos, localizados al noreste-sureste del cantón, de forma más expresiva hacia la ciudad de Montecristi. Este sistema de colinas compuestas tiene altitudes promedias de 200 msnm y están sobre un sustrato de rocas sedimentarias y volcánicas pertenecientes a unidades geológicas cretácicas y mesozoicas.

Podemos mencionar el relieve acolinado compuesto por las rocas de la Formación San Mateo en el Sector de La Sábana al oeste del Cerro Montecristi, el cual se caracteriza por relieves suaves con pendientes hasta de un 25\%, así como desniveles relativos menores a $25 \mathrm{~m}$. También se puede reconocer relieves acolinados bajos, localizados hacia el norte con el cantón Jaramijó, con cimas redondeadas que alcanzan desniveles relativos de hasta $25 \mathrm{~m}$. con vertientes convexas de pendientes dominantes del 12 al 25\%. También se reconocen relieves acolinados altos, con elevaciones que llegan a los $200 \mathrm{~m}$ de desnivel relativo, incluyendo pendientes hasta el 70\%. Constan así cimas agudas y vertientes rectilíneas, con una longitud de las vertientes que varían desde 50 a $250 \mathrm{~m}$. (cerro Jaboncillo).

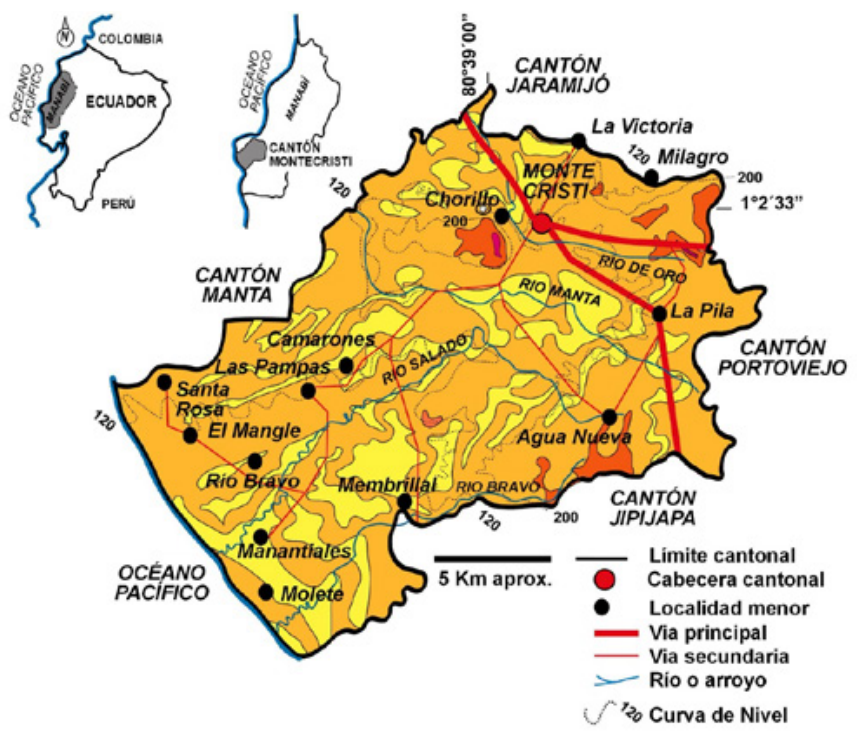

\begin{tabular}{|c|c|}
\hline $\begin{array}{c}\text { RANGOS DE } \\
\text { PENDIENTES CRITICAS }\end{array}$ & $\begin{array}{l}\text { CRITERIO GEOLÓGICO. } \\
\text { GEOTECNICO }\end{array}$ \\
\hline$<15^{\circ}(30 \%)$ & $\begin{array}{l}\text { ESTABLE TODO TIPO DE SUELO } \\
\text { Y ROCA }\end{array}$ \\
\hline $\begin{array}{c}15^{\circ}-30^{\circ} \\
(30 \%-65 \%)\end{array}$ & $\begin{array}{l}\text { INESTABLE ROCA Y SEDIMENTOS } \\
\text { DE GRANO FINO (ARCILLA-LIMO) }\end{array}$ \\
\hline $\begin{array}{l}>30^{\circ} \\
(>65 \%)\end{array}$ & $\begin{array}{l}\text { INESTABLE ROCA Y SEDIMENTOS } \\
\text { DE GRANO GRUESO (ARENAS - } \\
\text { GRAVAS) }\end{array}$ \\
\hline$>45^{\circ}(100 \%)$ & $\begin{array}{l}\text { MUY INESTABLE TODO TIPO } \\
\text { DE ROCA Y SUELO }\end{array}$ \\
\hline
\end{tabular}

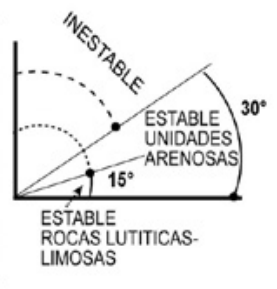

Figura 3. Rangos de pendientes críticas del cantón Montecristi. Fuente: Modificado de Cruden y Varnes (1996).
Las llanuras aluviales asociadas a colinas y lomas bajas aisladas, se reconocen en la parte media del cantón y zonas costeras, lugar donde se asienta la mayor parte de la población rural. Este sistema de colinas se hace menos frecuente hacia la parte norte del cantón, donde se localizan los asentamientos de Bajo Grande, Río de Cana, Río Seco y Santa Rosa, entre otros. Un aspecto importante de la localización de la ciudad de Montecristi es que allí se localizan las nacientes de las microcuencas principales de los ríos Manta y El Salado.

Los depósitos aluviales poseen una geometría alargada y no muy extensa, teniendo como eje los ríos Salado, la Caña, Bravo y Manta, con pendientes desde planas a muy suaves de $0 \%$ a $2 \%$ y de $2 \%$ al $5 \%$ y un desnivel relativo de 0 a $5 \mathrm{~m}$. En zonas bajas, se reconocen colinas con cimas planas y redondeadas, que alcanzan desniveles hasta $15 \mathrm{~m}$. con pendientes dominantes del $5 \%$ a $12 \%$.

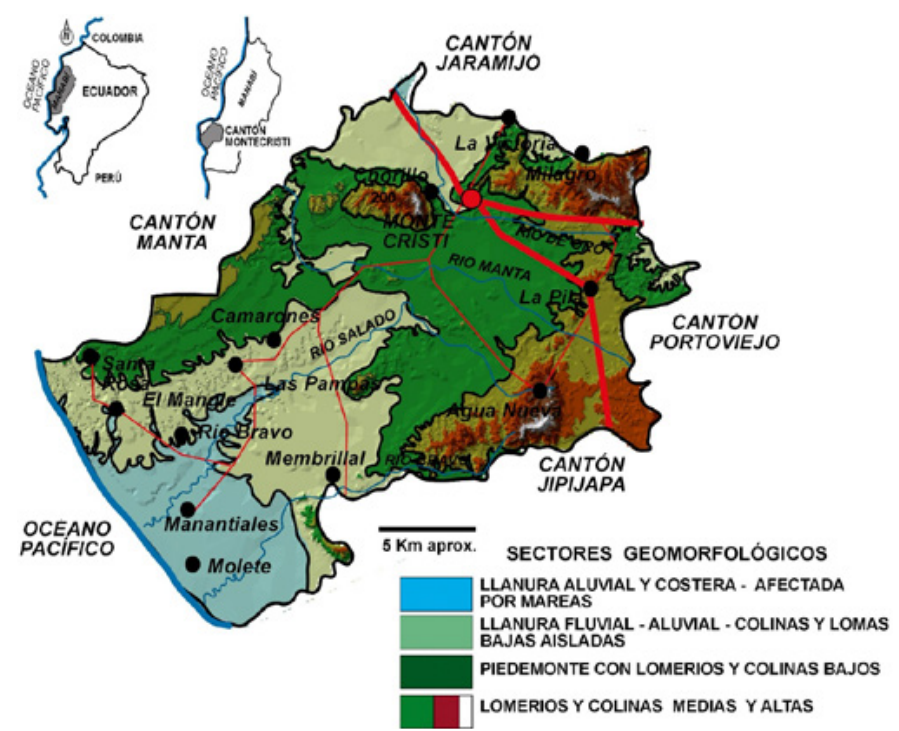

Figura 4. Sectores geomorfológicos dominantes del cantón Montecristi.

Llanura aluvial - costera afectada por marea, consiste en un complejo sedimentario que permite la construcción de paisajes costeros muy variados que definen al cantón como un territorio con "dos márgenes marinos"; el primero localizado hacia el norte (sector San Mateo - Manta), donde se forman sistemas de playas en bahía y el segundo, entre los sectores de Manta - San Lorenzo donde se forma barras playeras y acantilados costeros. De manera general, se reconocen formas de relieves tipo acolinado bajo con pendientes de $12 \%$ a $25 \%$, presentan desniveles menores a $15 \mathrm{~m}$.

\section{Aspectos climáticos e hidrográficos regionales}

Desde el punto de vista climático, se obtiene para el cantón un clima subhúmedo seco tropical con lluvias entre enero a marzo (Figura 5), pero debido a la alta temperatura constante (de media anual $24.5^{\circ} \mathrm{C}$ ), la tasa de evapotranspiración supera a las precipitaciones mensuales durante todo el año, produciendo un coeficiente de humedad negativo.

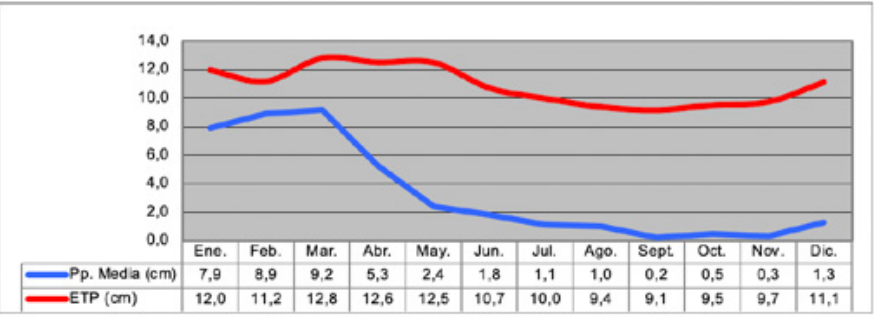

Figura 5. Balance hídrico para la localidad de Montecristi.

Fuente: Datos estación M0445 Los Cerros -Montecristi INAMHI (2017). 
Esta condición climática produce, junto con el factor orográfico del cantón, pisos bioclimáticos con tipo arbustal espinoso hacia la vertiente seca, donde se asientan la población de Montecristi y en las localidades del litoral costero, mientras que hacia la vertiente húmeda se puede encontrar una variedad entre selva húmeda y caducifolia (Figura 6).

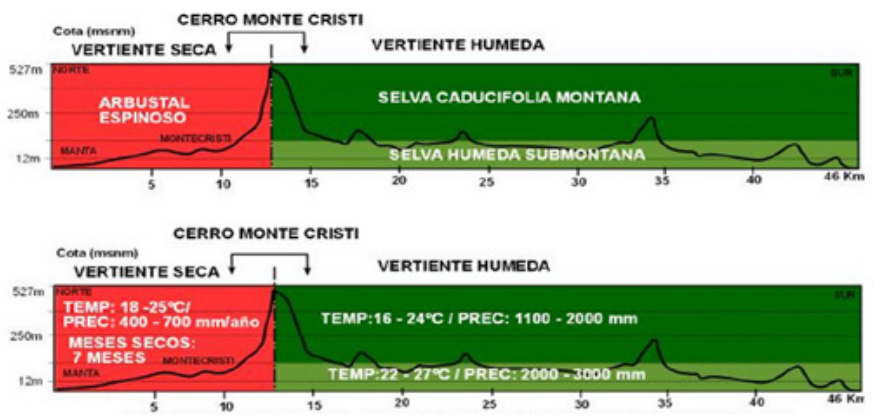

Figura 6. Condiciones climáticas y ecológicas generales para el cantón Montecristi zonas aledañas. Fuente: Datos INAMHI (2017).

\section{Morfometría de las microcuencas hidrográficas del cantón Montecristi}

En cuanto al sistema hidrológico, se puede reconocer tres grandes microcuencas hidrográficas: en primer lugar, la microcuenca del río Bravo (Figura 7), la cual se extiende desde el sureste al noroeste del cantón, a una altitud de $340 \mathrm{msnm}$ en dirección hacia el océano Pacífico, que se caracteriza por tener un patrón dendrítico denso y anular en la subcuenca media y alta, mientras que hacia la subcuenca baja el río tiene geometría meandriforme con valor de sinuosidad (IS $<0.64$ ) y pendiente $<0,5 \%$.

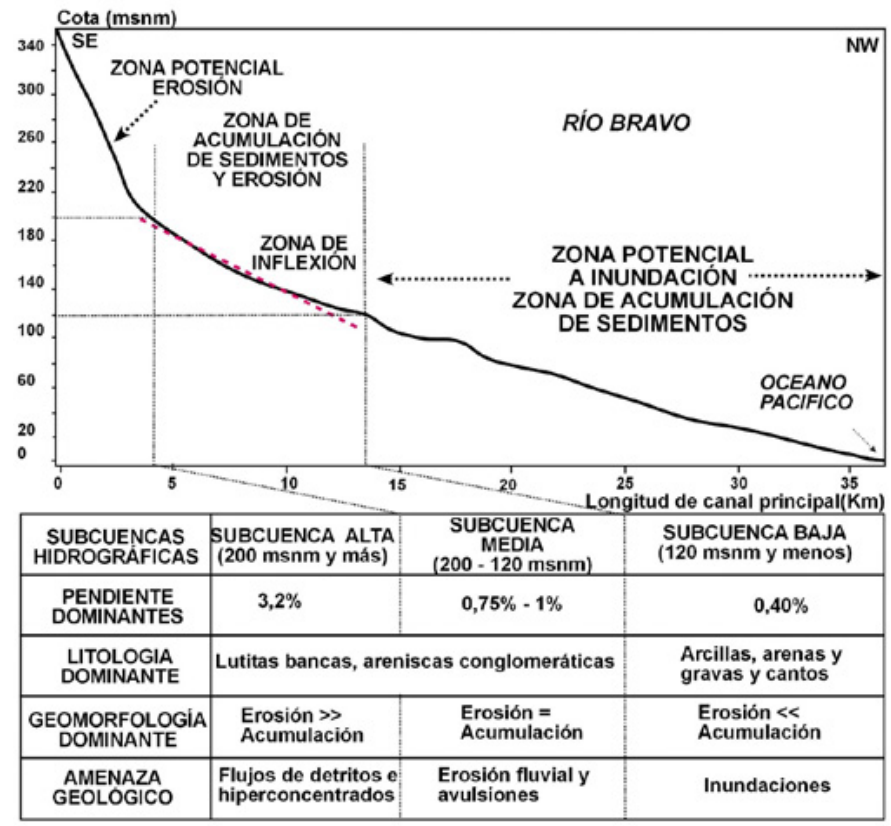

Figura 7. Perfil de equilibrio de la sección fluvial del Río Bravo y distribución de subcuencas hidrográficas.

En segundo lugar, existe la microcuenca del río Salado (Figura 8), que recorre la región central del cantón desde el sureste a noroeste y se inicia a 520 msnm, en dirección hacia el océano Pacífico; se caracteriza por tener un patrón dendrítico medio a denso y rectangular, controlado por fallas geológicas locales en la subcuenca media y alta, mientras que hacia la subcuenca baja tiene geometría meandriforme con valor de sinuosidad (IS $<0.63$ ) y pendiente $<0,5 \%$.

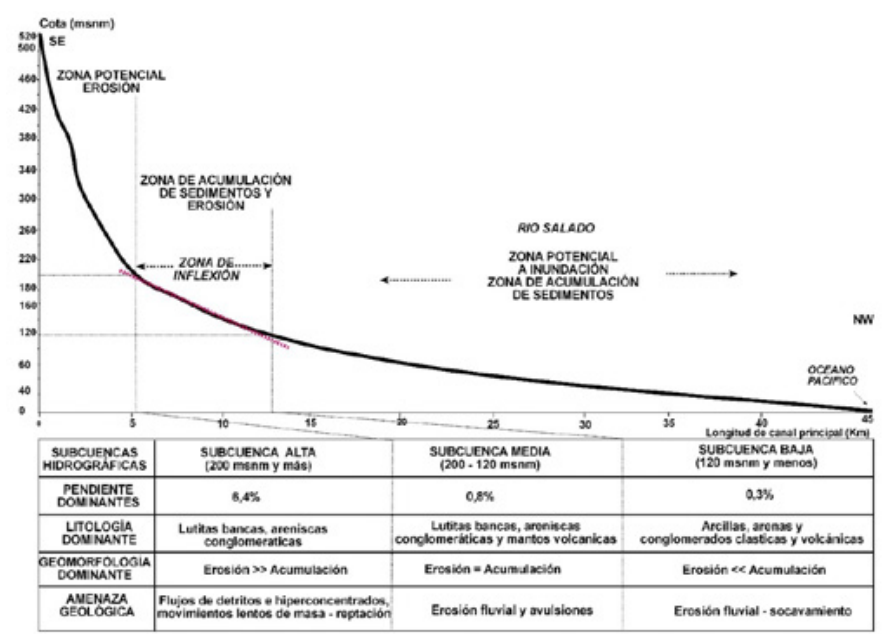

Figura 8. Perfil de equilibrio de la sección fluvial del Río Salado y distribución de subcuencas hidrográficas.

Finalmente, la microcuenca del Río Manta (Figura 9) se extiende en la sección sur del cantón; se inicia a 240 msnm y desemboca en el océano Pacífico. Tiene un patrón mixto desde dendrítico denso, anular y rectangular, especialmente en la subcuenca media y alta, mientras que hacia la subcuenca baja, el río se reactiva debido a cambios de pendientes. Forman una segunda zona de inflexión, producto del efecto del anticlinal, con un drenaje tendente a formar geometría recta y trenzada con valor de sinuosidad (IS < 0.74) y pendiente de $1.5 \%$.

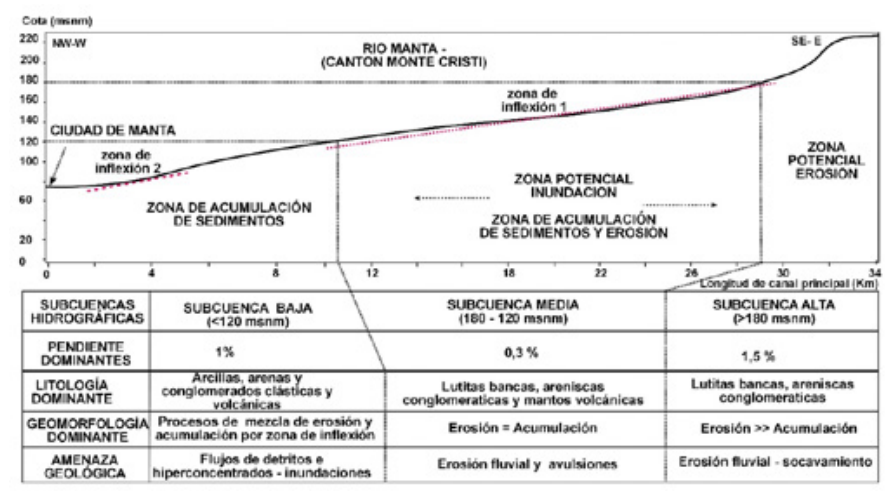

Figura 9. Perfil de equilibrio de la sección fluvial del Río Manta y distribución de subcuencas hidrográficas.

\section{Amenaza por inundación}

En virtud de todo lo analizado, se puede afirmar que el cantón Montecristi es un territorio irregular y, debido a ello, se ha identificado sectores que sufren movimientos de masas e inundaciones constituyéndose así en zonas vulnerables. En la temporada de lluvias del 2015, por ejemplo, se dio un represamiento de aguas, al no existir un adecuado sistema de drenaje, lo que provocó inundaciones, colapso de puentes por azolvamiento del cauce, ocasionando pérdidas económicas cuantiosas (GAD Municipal Montecristi, 2016).

Después de valorar la morfometría de las microcuencas hidrográficas, se estima un balance hídrico negativo, con evapotranspiración media anual de $1306 \mathrm{~mm}$ y precipitaciones media anual de $399 \mathrm{~mm}$, lo cual produce un coeficiente de humedad de $-86 \mathrm{~mm}$ y un índice hídrico anual de $-416 \mathrm{~mm}$. Por tal motivo, las microcuencas solo pueden desarrollar inundaciones por lluvias excepcionales.

Según MAG (2017), a partir de los resultados obtenidos en estudios de las cuencas de los ríos Caña, Manta, Portoviejo y Bravo (Tabla 1), se obtiene 
un módulo especifico de caudal comprendido entre 1 - $10 \mathrm{l} / \mathrm{s} / \mathrm{km} 2$; por consiguiente, las microcuencas del cantón Montecristi corresponde a caudales medio que no supera el índice hídrico anual de $416 \mathrm{~mm}$.

Tabla 1. Índice de compacidad de microcuencas hidrográficas.

\begin{tabular}{|c|c|c|c|}
\hline $\begin{array}{c}\text { Microcuen- } \\
\text { cas hidro- } \\
\text { gráficas }\end{array}$ & $\begin{array}{l}\text { Índice de } \\
\text { compacidad }\end{array}$ & $\begin{array}{l}\text { Forma de la micro- } \\
\text { cuenca }\end{array}$ & $\begin{array}{l}\text { Tendencia a } \\
\text { crecidas }\end{array}$ \\
\hline Río Salado & 1.20 & \multirow{2}{*}{$\begin{array}{l}\text { Redonda a oval redon- } \\
\text { deada }\end{array}$} & \multirow{2}{*}{ Media } \\
\hline Río Bravo & 1.17 & & \\
\hline Río Manta & 1.65 & $\begin{array}{l}\text { Oval oblonga a rectan- } \\
\text { gular oblonga }\end{array}$ & baja \\
\hline
\end{tabular}

Fuente: MAG (2017).

La sectorización de inundaciones (Figura 10) para lluvias excepcionales queda distribuida de la siguiente manera: en primer lugar, zona de susceptibilidad alta a inundación corresponde con el área por debajo de la cota $120 \mathrm{msnm}$ (incluye la parte media y baja del cantón y las subcuencas bajas de los ríos Bravo, la Caña y Salado). Seguidamente, zona con susceptibilidad media (corresponde las áreas entre las cotas 120 msnm y 200 msnm), y finalmente la zona con susceptibilidad baja, que corresponde con la cota $200 \mathrm{msnm}$ y superiores.

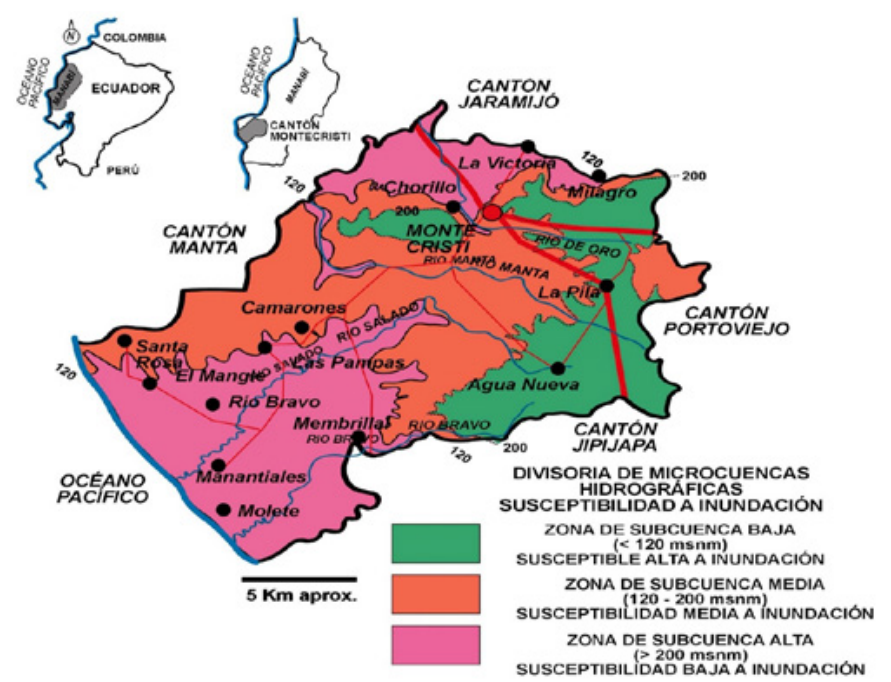

Figura 10. Divisoria de las microcuencas del cantón Montecristi y su potencialidad a inundaciones

Según inventario de impacto de crecida realizado por el GAD cantón de Montecristi (2016) para un total de 18917 viviendas, se registran 1317 (7\%) localizadas en zonas con elevada vulnerabilidad a inundaciones; 1550 edificaciones (8\%) se encuentran en vulnerabilidad alta a media y $2661(11 \%)$ en vulnerabilidad baja. El restante de edificaciones, un total de 13.389 (74\%) se encuentra en condición vulnerable muy baja. Por tanto, se considera que la mayoría de las viviendas se encuentra en zona con baja a muy baja susceptibilidad a riesgos de inundación.

\section{Evaluación geomecánica de suelos en la ciudad de Montecristi.}

En base a los datos de perforación de suelos de la ciudad de Montecristi (Figura 11), no se registra nivel freático en los perfiles de suelos hasta $10 \mathrm{~m}$. de profundidad y bajos espesores de sedimentos, indicando que la roca se localiza a 4 metros de profundidad. Por otro lado, hacia el sector sur de Montecristi, los suelos son más espesos y llegan a alcanzar $10 \mathrm{~m}$. La ciudad de Montecristi está localizada sobre suelos esencialmente aluviales y residuales arenosos, con propiedades geomecánicas friccionantes y debe ser considerado por ende un terreno potencial a licuación. Mientras las zonas al sur del cantón son más arcillosas, el material granular del suelo tiene propiedades cohesivas y son potenciales a reptación en zonas con pendientes mayores a $15^{\circ}$.

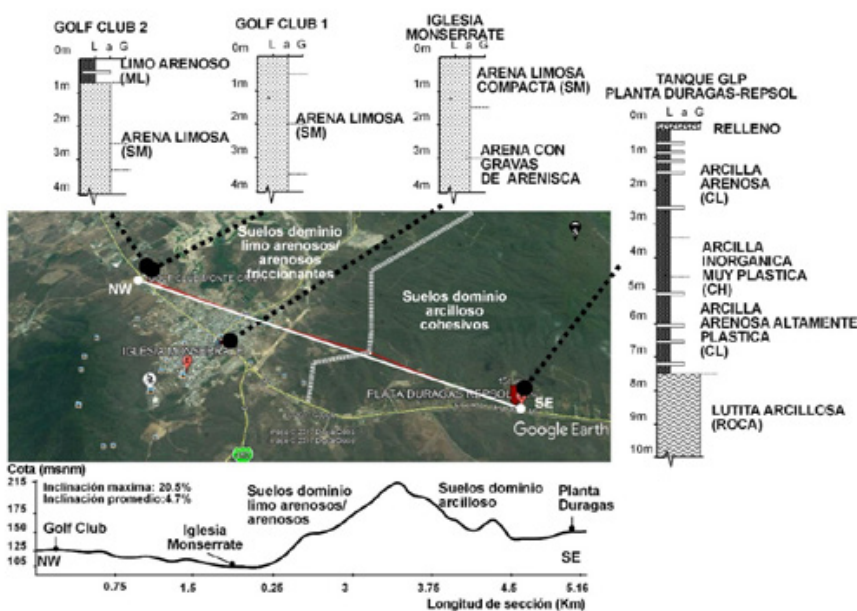

Figura 11. Distribución de perfiles de suelos en varias localidades de la ciudad de Montecristi y zona aledaña.

Fuente: INGENNYA - NYLIC (2013). Imagen Google Earth, (2017)

Los resultados de la integración de los datos permitieron reconocer cuatro grandes zonas con comportamientos de suelos geomecánicos diferentes (Figuras 12 y 13); suelos de material granular fino (arcilla) hacia la zona baja de la ciudad de Manta (cotas $<10 \mathrm{msnm}$ ), que incluye influencia de material sedimentario proveniente del mar a profundidades mayores 8-10m, con restos de materia orgánica y restos fósiles de conchas marinas. De manera general, se reconoce niveles freáticos entre 3 y 10 de profundidad, así como espesas secuencias de sedimentos finos (arcillas y limos) hacia la cota de $7 \mathrm{msnm}$, mientras que hacia la cota de $10 \mathrm{msnm}$ y más alejados de la línea de costa se reconocen importantes contenidos de estratos arenosos.

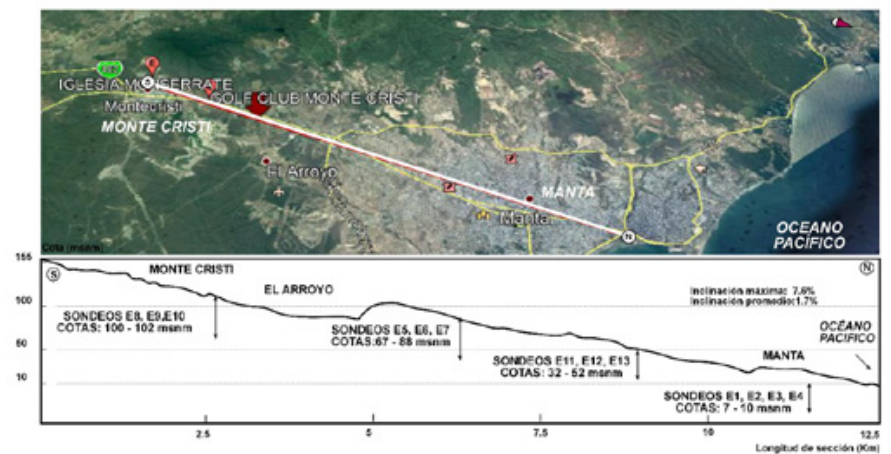

Figura 12. Distribución de los sondeos geomecánicos de la sección Montecristi - Manta. Fuente: Imagen Google Earth (2019).

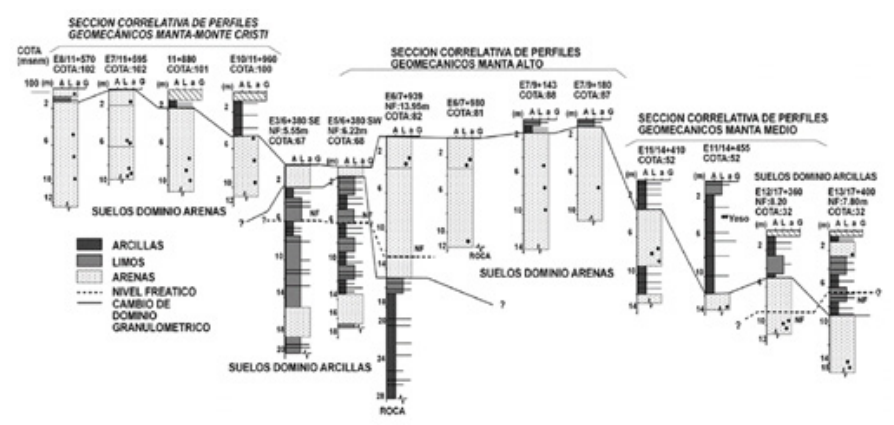

Figura 13. Correlaciones de geomecánica de suelos para secciones de la ciudad de Manta media, alta y en la ciudad de Montecristi. Fuente: Datos INGENNYA - NYLIC (2013). 
Hacia la zona de la ciudad de Montecristi y Manta Alto, se tienen espesores de suelos arenosos de hasta $12 \mathrm{~m}$. Estos sectores están expuestos a fenómenos de licuación de suelos, debido a su homogeneidad granuIométrica y a la cercanía a fuentes sísmicas activas del Pacífico, por lo que se recomienda disminuir los niveles freáticos para mitigar el impacto que ocasionaría un sismo. La zona que presenta la mejor calidad geomecánica de suelos se reconoce en la sección de Manta Medio (cotas 10 - 50 msnm), debido a mezcla de material friccionantes (arena - limo) y cohesivo (arcillas).

\section{Amenaza sísmica}

Según SNGRE (2017), en la región de Manta - Montecristi, la sismicidad durante el siglo XX cae dentro de la gama de magnitudes medias $(\mathrm{Mw}<$ 7.1). El registro instrumental muestra la existencia de varios "enjambres" sísmicos para los años 1998, 2002 y 2005 (Vaca et al., 2007). El "enjambre" sísmico del 2005 caracteriza por la ocurrencia de cuatro sismos Mw > 6, 11 sismos con Mw entre 5 y 5.9 y alrededor de 470 sismos, con una magnitud Mb entre 4 y 4.9 (SNGRE, 2017; ONU - PNUD, 2017). La alta peligrosidad sísmica de la zona costera del país se produce por la subducción de la Cordillera de Carnegie y los sistemas de fallas geológicas activas a lo largo de toda la costa, principales fuentes sísmicas del Pacífico ecuatoriano (Figura 14).

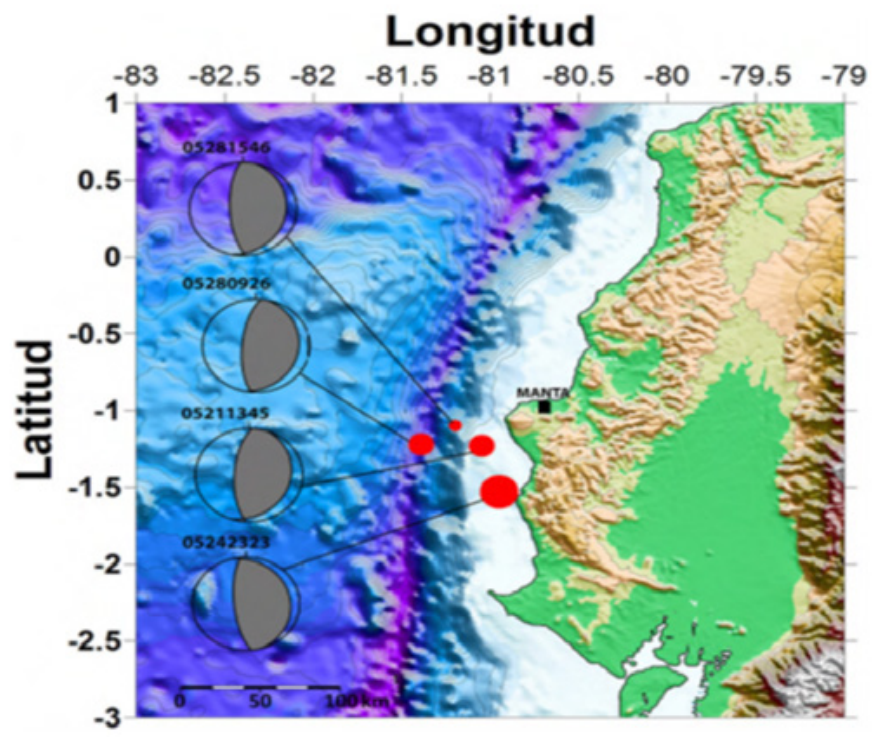

Figura 14. La zona de subducción ubicada en el océano Pacifico, la cordillera submarina de Carnegie y su contacto con la placa Suramericana (Tomado de Vaca et al., 2007).

El informe del GAD (2016), sobre tipo de edificaciones afectadas por actividad sísmica en el cantón Montecristi, registra 19015 viviendas, de las cuales $41(0.22 \%)$ se localizan en zonas de vulnerabilidad sísmica muy alta. 12845 de ellas (67.92\%) están expuestas a vulnerabilidad sísmica alta y 4146 (21.92\%) se encuentra en vulnerabilidad baja. El restante de edificaciones 1882 (9,95\%) se encuentra en zonas con muy bajo susceptibilidad ante este evento.

El cantón está afectado por un conjunto de fallas geológicas (Figura 2 y Tabla 2), que surcan el territorio en el sentido noreste - suroeste, siendo su potencial sísmico calculado según varios procedimientos geofísicos que se indican a continuación:
Tabla 2. Datos de mediciones sobre potencial de magnitud y área de influencia sísmica de fallamiento local.

\begin{tabular}{lllllll}
\hline $\begin{array}{l}\text { Pará- } \\
\text { me- } \\
\text { tros }\end{array}$ & $\begin{array}{l}\text { Longitud } \\
(\mathrm{km})\end{array}$ & $\begin{array}{l}\text { Mag- } \\
\text { nitud } \\
(\mathrm{m})\end{array}$ & $\begin{array}{l}\text { Mo- } \\
\text { mento } \\
\text { sísmico } \\
(\mathrm{Mo})\end{array}$ & $\begin{array}{l}\text { Magni- } \\
\text { tud del } \\
\text { momento } \\
(\mathrm{Mw})\end{array}$ & $\begin{array}{l}\text { Área } \\
(\mathrm{km} 2)\end{array}$ & $\begin{array}{l}\text { Despla- } \\
\text { zamiento } \\
\text { cosísmico } \\
(\mathrm{m})\end{array}$ \\
\hline $\begin{array}{l}\text { Falla } \\
\text { (A) }{ }^{*}\end{array}$ & 21,9 & 7,1 & $\begin{array}{l}1 \times 10^{26} \\
\text { dynas/ } \\
\text { cm }\end{array}$ \\
Falla & 15,1 & 6,8 & 1100 & 1,2 \\
(B) & 6,8 & $\begin{array}{l}2 \times 10^{25} \\
\text { dynas/ } \\
\mathrm{cm}\end{array}$ \\
Falla & 8,6 & 6,3 & 125 & 1,05 \\
(C) & 6,4 & $\begin{array}{l}3 \times 10^{25} \\
\text { dynas/ } \\
\mathrm{cm}\end{array}$ \\
\hline
\end{tabular}

Fuente: Gómez y Salcedo (1987). * Corresponde con la Figura 2.

La Falla A (Figura 2) que afecta a Montecristi tiene un potencial estimado de magnitud Mw 7.1 y 6.8, afectando un radio de área de al menos $1.100 \mathrm{Km} 2$, con importante desplazamiento cosísmico a lo largo de la traza de falla. Por otro lado, las fallas geológicas $B$ y $C$ afectan el sector La Pila; el potencial es de M 6,4-6,8 y Mw 6,3-6,1, con zona de radio de afectación de $125 \mathrm{Km} 2$.

El 16 de abril de 2016 ocurrió un evento sísmico a las 18:58 ECT (SNGRE, 2019), con epicentro entre las parroquias Pedernales y Cojimíes del cantón Pedernales, en la Provincia de Manabí, con una magnitud de 7,8 Mw, uno de los más destructivos desde el terremoto de 1987. Según datos del SNGRE (2017) y ONU-PNUD (2019), en el cantón Montecristi se registraron daños considerables, especialmente en la Comuna "Las Lagunas" de la Parroquia rural La Pila, denominada la "Zona 0" del cantón, debido al alto número de viviendas y familias afectadas.

\section{Amenaza a movimientos de masa del terreno- condiciones geotécnicas}

EI GAD (2016) y SNGRE (2019) realizaron sendos informes donde evalúan la susceptibilidad a eventos de movimiento en masa del Cantón para un total de 63262 habitantes. 702 habitantes (1.11\%) se ubican en zonas de vulnerabilidad muy alta a movimientos de masa; 4787 hab. (8\%) se encuentra en zonas de vulnerabilidad alta a media y 7269 hab. (11\%) se encuentra en vulnerabilidad baja. El restante de la población (46776 hab., el 74\%) se encuentran en zonas de muy baja y sin susceptibilidad ante este evento.

El cantón presenta movimiento de masa del terreno (Figura 15) distribuido como; zonas geológico-geotécnicas muy estables a estables, relacionadas con pendientes muy bajas a bajas $\left(<15^{\circ}\right)$ que ocupan la mayor proporción territorial, donde se localizan las zonas urbanas más pobladas. 


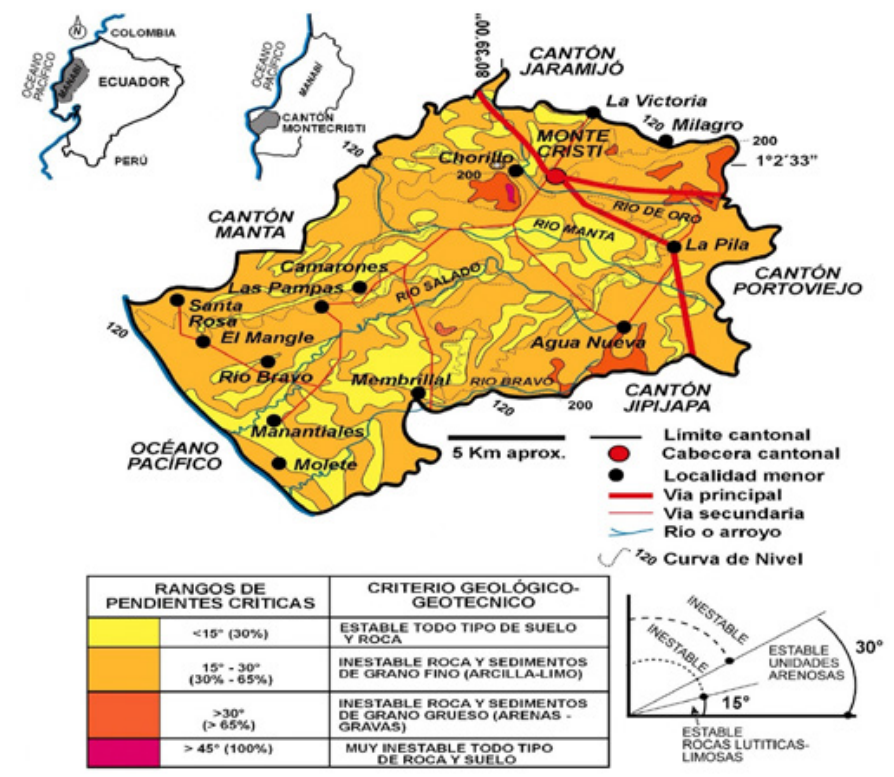

Figura 15. Distribución de zonas con riesgo geológico - geotécnico del Cantón Montecristi. Fuente: Modificado de Cruden y Varnes (1996).

Zonas geológico-geotécnicamente inestables se ubican en la ya mencionada localidad de "La Pila", donde se registran pendientes entre $15^{\circ}$ $30^{\circ}$, asociadas a suelos y rocas de composición arcillo-limosos y las zonas inestables; se distribuyen de manera dispersa en el Cantón, con geoformas de colinas y lomas altas (pendientes $>30^{\circ}$ ) compuestas por rocas y suelos de tipo arcillo-limosos.

\section{CONCLUSIONES}

El cantón Montecristi es dominado por unidades geológicas sedimentarias y volcánicas, deformadas por pliegues estructurales que forman sinclinales en la zona central del cantón y alineamiento de la microcuenca del río Salado - La Caña.

Se reconocen zonas con susceptibilidad alta a inundación en todas las áreas por debajo de la cota $120 \mathrm{msnm}$. La susceptibilidad media se ubica entre las cotas 120 msnm y 200 msnm, y con baja susceptibilidad corresponde al territorio por encima de las cotas 200 msnm. La amenaza por movimientos de masa divide al cantón en, por un lado, zonas geológico-geotécnicas muy estables y estables (lo que representa la mayor proporción territorial), zonas inestables (localizadas mayoritariamente en el sector La Pila) y finalmente zonas muy inestables, que se distribuyen de manera dispersas en el cantón.

La amenaza sísmica es la más importante dentro del cantón, debido a su cercanía al eje de subducción del Pacifico y al fallamiento local con actividad neotectónica. Se recomienda las edificaciones de baja altura con fundaciones y cálculos ajustados a zonas sísmicas (Zona VI, valor Z $\geq 0.50$ ) con suelos licuables, así como elaborar la microzonificación sísmica urbana.

\section{REFERENCIAS BIBLIOGRÁFICAS}

American Society for Testing and Materials -ASTM (2001). Unified Soil Classification System - SUCS and AASTHO (American Association of State Highway and Transportation Officials. 395-408 pp.

Banco Interamericano de Desarrollo - BID (2019). Resumen ejecutivo de la metodología de la evaluación de Desastres y Cambio climático. Documento técnico. División de cambio climático. Unidad de Salvaguardias ambientales y sociales. Series VIII.
Camargo Mora, M. G. (2010). Operacionalización del Plan de Desarrollo Urbano Local (PDUL): Fundamentos de la planificación.Vol. 51. Mérida, Mérida, Venezuela: Revista Geográfica Venezolana. Universidad de Los Andes.

Cruden, M., y Varnes, D. (1996). Landslide Types and Processes, Transportation Research Board, U.S. National Academy of Sciences, Special Report, 247, (247), 36-75. Retrieved from https://www.researchgate.net/publication/269710331\%0ACruden,D.M.,Varnes,

GAD - Gobierno Autónomo Descentralizado Municipal del cantón Montecristi (2016). Actualización plan de desarrollo y ordenamiento territorial 2016, con énfasis en gestión de riesgos. Diagnóstico estratégico. 526 p.

Gómez, A. y Salcedo, E. (1987). Leyes de Atenuación de la intensidad Macrosísmicas en Colombia. Istituto Nazionale di Geofisica e Vulcanologia sezione di Milano, 15.Italia.

Guerrero, 0., y Benavides, M. (2020). Gestión de riesgos de desastres y ciudades sostenibles.

VI Seminario Iberoamericano de Desarrollo, Sostenibilidad y Ecodiseño. La sostenibilidad en tiempo de Covid 19. ULA - UPV. España Venezuela.

Instituto Nacional de Meteorología e Hidrología - INAMHI (2017): Anuario Meteorológico 1963-2013. Quito - Ecuador

Instituto Nacional de Estadísticas y Censos - INEC (2010). Estadísticas y Proyecciones. Recuperado de: https://www.ecuadorencifras. gob.ec/institucional/home/.

INIGEMM (2020). Mapa geológico mínero de la Provincia de Manabí y del Cantón Montecristi. Consultado en: http://historico.mineria. gob.ec/inigemm-presenta-la-actualizacion-del-mapa-geologico-del-ecuador-en-expominas-2017/. Fecha de consulta: 01/09/2020.

INGENNYA - NYLIC (2013). Estudio geológico geotécnico de la sección vial Montecristi - Manta. Ecuador. Informe técnico inédito. 87p.

Loor, M. (2015). Participación ciudadana y Gestión del riesgo, caso: Parroquia urbana Leónidas Proaño del cantón Montecristi. Pontificia Universidad Católica del Ecuador, Escuela de Trabajo Social. Magíster en gestión del desarrollo local comunitario. Quito-Ecuador.

MAG (2017). Generación de Geoinformación para la gestión de territorio a nivel nacional. Escala 1:25.000. Cantón Manta. Componente 3: Cima e Hidrología. Memoria Técnica. Ecuador.

Normas ecuatorianas de la Construcción - NEC-11 (2019). Zonificación Sísmica del Ecuador. Conultado:https://www.researchgate.net/ publication/303256522_Analisis_Sismico_de_Estructuras_ con_Disipadores_de_Energia_ADAS_0_TADAS/figures? $10=1$ (Consulta 01de Febrero 2020).

ONU - PNUD (2017). Search Results for: Desastres naturales. Consulta: http://onu.org.gt/?s=desastres+naturales (Consultado 15 de febrero 2020).

Planifica Ecuador (2019). Propuesta metodológica para la planificación prospectiva territorial de los gobiernos autónomos descentralizados. Secretaría Técnica Planifica Ecuador, julio 2019, 40p.

PMA, GCA (2009). Atlas de Deformaciones Cuaternarias de los Andes, Servicio nacional de Geología y Minería, Publicaciones Geológicas Multinacionales, № 7, 320p

Servicio Nacional de Gestión de Riesgos y Emergencias - SNGRE (2019). Lineamientos para incluir la gestión del riesgo de desastres en el Plan de Desarrollo y Ordenamiento Territorial (PDOT) julio 2019. Quito - Ecuador. 80p.

Vaca, S., Régnier, M., Methoux, N., Alvarez, V., y Pontoise, B. (2007). Sismicidad de la región de Manta: Enjambre sísmico de Manta-2005. IG-EPN, Quito, Ecuador UMR GéoAzur, IRD - Université de Nice-Sophia-Antipolis, France. 